\title{
Administration of metformin in renal transplant patients with post-transplant diabetes mellitus
}

\author{
Masoumeh Asgharpour ${ }^{1}$, Seyed Hadi Mirhashemi ${ }^{2}$, Amirhesam Alirezaei $^{*} \mathbb{1}$ \\ ${ }^{1}$ Department of Nephrology, Rouhani Hospital, Babol University of Medical Sciences, Babol, Iran \\ ${ }^{2}$ Department of Surgery, Loghman hakim Hospital, Shahid Beheshti University of Medical Sciences, Tehran, Iran \\ ${ }^{3}$ Urology and Nephrology Research Center, Shahid Modarres Hospital, Shahid-Beheshti University of Medical Sciences, Tehran, Iran
}

\section{A R T I C L E I N F O}

Article Type:

Mini-Review

\section{Article History:}

Received: 2 April 2019

Accepted: 10 June 2019

Published online: 30 June 2019

\section{Keywords:}

Post-transplant diabetes mellitus, New-onset diabetes after transplantation, Diabetes mellitus, Transplantation,

Immunosuppressive drugs, Obesity

\begin{abstract}
A B S T R A C T
Diabetes is the most common cause of renal failure which is frequently seen in candidates of solid organ transplant both before and after of the transplantation. For successful long-term tissue graft, it is important to control blood glucose level particularly after transplantation. Post-transplant diabetes mellitus (PTDM) is the main issue contributing to cardiovascularrelated mortality in kidney transplant recipients. Important risk factors of PTDM include using immunosuppressive drugs, post-transplant weight gain and obesity, and the presence of pre-transplant diabetes. Because of safety concerns, there has been a consensus from 2003 onward to cease metformin as the first-line anti-diabetic drug in patients with PTDM. The relationship between PTDM and metformin administration in high-risk renal transplant patients needs to be validated by more trial studies to establish the risk-benefit balance using this drug. Here, we reviewed the pros and cons of using metformin by presenting conclusions from several retrospective and clinical trial studies.
\end{abstract}

Implication for health policy/practice/research/medical education:

Metformin is a safe and tolerable drug within one year after renal transplantation encouraging clinicians to administer this drug to renal transplant candidates.

Please cite this paper as: Asgharpour M, Mirhashemi SH, Alirezaei A. Administration of metformin in renal transplant patients with post-transplant diabetes mellitus. J Renal Inj Prev. 2019;8(3):190-194. DOI: 10.15171/jrip.2019.35.

\section{Introduction}

In patients with renal failure, kidney transplantation has boosted the survival and quality of life and decreased expenses on time compared to dialysis therapy. Compared with normal population; nevertheless, graft recipients are at higher risk of death in particular secondary to cardiovascular events as the causes of half of all deaths in these patients.

\section{Diabetes and transplantation}

Diabetes is the most common cause of renal failure and is frequently seen in patients with solid organ transplant both before and after transplantation. Transplantation candidates with diabetes have had augmented risk of cardiovascular diseases and inflammation compared with nondiabetic individuals undergoing solid organ transplantation.
New-onset diabetes after transplantation (NODAT)

For durable and successful grafts, it is of critical importance to monitor and control blood glucose level particularly after transplantation. The NODAT seems to be the main culprit for cardiovascular related death in kidney transplant recipients. Diabetes is frequently diagnosed in transplant patients both before and after the transplantation. In a study published in 2018, undiagnosed diabetes was detected in $8 \%$ of organ recipients using glucose tolerance test. The term post-transplant diabetes mellitus (PTDM) has recently been proposed as an alternative terminology for the NODAT (1). PTDM as a prevalent condition inflicts up to $50 \%$ of renal transplanted patients and augments the risks of cardiovascular diseases, graft and mortality. In fact, poor controlling of blood glucose level after renal transplantation has been related to adverse transplant outcomes. Because of higher risk 
of impaired function of allograft in diabetic transplanted patients, pharmacological intervention is necessary to manage PTDM in these individuals (2). Administration of immunosuppressive drugs, which deregulating glucose metabolism, has been interpreted as one of the risk factors for PTDM. In fact, immunosuppressive regimens prescribed in transplanted patients may interfere with the functions of pancreatic beta-cells and insulin secretion which result in poor regulation of blood glucose level. The other risk factors of PTDM consisted of post-transplant weight gain and obesity, especially during the first year after transplantation. Post-transplant overweight has been related to $10-35 \%$ of insulin resistance cases in transplanted patients. Another important risk factor of PTDM has been the presence of pre-transplant diabetes (3).

\section{Materials and Methods}

For this mini-review, we used a variety of sources including PubMed, Web of Science, Embase, EBSCO, Google Scholar and Scopus. The search was conducted by using combinations of the following key words and or their equivalents; post-transplant diabetes mellitus, insulin resistance, new-onset diabetes after transplantation, diabetes mellitus, transplantation, immunosuppressive drugs, obesity, cardiovascular diseases, inflammation, metformin, lactic acidosis, end-stage renal disease, cancer, liver failure, renal transplantation, kidney failure, nephroprotection, glomerular filtration rate, nephrotoxicity ,pre-transplant diabetes and AMPactivated protein kinase (AMPK).

\section{Pharmaceutical management of PTDM}

Glucose metabolism is dysregulated in renal-transplanted patients. Decreased insulin secretion after renal transplantation seems to be the main pathological factor. Therefore, therapeutics that primarily modify the function of pancreatic beta-cells are theoretically beneficial in these patients (4). In patients with stable allograft function (estimated glomerular filtration rate; eGFR $>30 \mathrm{ml} / \mathrm{min}$ ), metformin has been traditionally the first line therapy for managing PTDM early after transplantation. However, in later phases after transplantation, the NODAT agreement in 2003 did not endorse metformin as the first-line anti-diabetic drug because of safety concerns. In later phases after transplantation, sulfonylureas, meglitinides, dipeptidyl peptidase-4 inhibitors, alpha-glycosidase inhibitors, and rarely sodium-glucose co-transporter 2 (SGLT-2) inhibitors have been suggested as potential anti-glycemic agents $(5,6)$. There is a need for further trial studies to verify an association between PTDM and metformin administration in high-risk renal transplant patients and to evaluate the risk-benefit balance using this drug (7).

\section{Advantages of metformin}

It has been revealed that metformin decreases the incidence of vascular complications without being associated with the risk of weight gain or hypoglycemia in diabetic patients. In addition to its anti-cancer characteristics, metformin can also improve the signs of metabolic syndrome such as insulin resistance and inflammation. Probable metformin functional mechanisms consist of nephroprotection (8), anti-inflammation and immune-modulatory effects via activation of AMP-activated protein kinase (AMPK) and its downstream signal transducer and activator of transcription 3 (STAT3), and prevention of pancreatic beta-cells (9) and regulatory $\mathrm{T}$ cells (10) differentiation. AMPK, an enzyme involved in the pathogenesis of inflammation, has been a protective contributor against PTDM development. Overall, metformin acts as an antidiabetic agent by decreasing beta-cell dysfunction at early post-transplant period (11). Metformin may decrease nephrotoxicity through mitochondrial-dependent pathways as revealed in clinical models exposed cisplatin or gentamicin.

Metformin-based regimes have been noted to reduce the risk of graft rejection; nevertheless, these associations have not been statistically significant. Metformin has also been effective in preventing or delaying the progression of prediabetes to diabetes in at-risk individuals. Metformin is generally known as an efficient, safe, and affordable drug. Nevertheless, there is a need for more investigations to show whether or not such advantages could be extrapolated from general population to high-risk candidates of kidney transplantation. Experimental models propose that metformin decreases immunosuppressant-induced diabetes and improves glucose regulation by decreasing cell death (12). In one study on patients with PTDM; it was indicated that the selection of anti-hyperglycemic agents should be based on the type of immunosuppressant regimens. In this regard, metformin may be indicated in graft recipients under sirolimus but not tacrolimus based immunosuppressive therapy. Management of PTDM based on the immunosuppressant regimens offers a new strategy for treating this condition (13). Furthermore, metformin has shown an excellent safety profile in combination with other immunosuppressive and anti-diabetic drugs.

Renal transplanted patients are at higher risk of cancer up to three times than the general population. It has been shown that metformin reduces the risk of cancers and cancer-related death (14). Although the experimental and clinical evidence are insufficient, metformin has been suggested as the first-line anti-diabetic drug in transplanted patients. Nevertheless, additional studies are needed in this area (15).

\section{Potential complications of metformin}

Regarding concerns about the tolerability and safety of metformin in transplant candidates who are under complex multidrug regimens, clinicians are generally cautious in prescribing this drug. 
Some of potential complications of metformin

Lactic acidosis

A major concern discouraging clinical experts to use metformin is the risk of lactic acidosis which hypothetically can be amplified following metformin administration in transplant candidates. Although the risk of metformininduced lactic acidosis has been insignificant in general population, the presence of associated comorbidities may exaggerate the risk of this phenomenon. Nevertheless, there is no evidence that metformin increases the risk of lactic acidosis in the absence of other comorbidities. On the other hand, an exaggerated risk of lactic acidosis has been described in the presence of heart, renal and liver disorders even without using metformin. There has been no association between the plasma levels of lactate and metformin in other reports proposing the drug as a harmless bystander. In usual doses, metformin may even be a protective agent against lactic acidosis (11). There may be; however, an exaggerated risk of lactic acidosis in organ transplant candidates with kidney damage and drug-induced nephrotoxicity (16).

The Food and Drug Administration (FDA) recommends the withdrawal of metformin following hospitalization, and in patients with heart, renal or liver failure. These withdrawal recommendations are likely to be indicated at early post transplant phase because of higher risk of metformin complications at this time period $(17,18)$.

In a comprehensive systematic review examining 347 trials with 70000 patient-years of metformin use, no case of lactic acidosis and no significant association between the blood lactate levels and metformin administration were recognized (19). A higher risk of lactic acidosis has been reported in patients using sulfonylureas in comparison with metformin (20). On the other hand, patients with serum creatinine levels below than $1.5 \mathrm{mg} /$ $\mathrm{dL}$ have shown no increased risk of lactic acidosis (21). In a study on 2000 Korean diabetic patients, tissue hypoxia was accompanied with lactic acidosis and increased plasma lactate while no association was reported with metformin therapy (22). However, the rate of renal excretion of metformin meaningfully declined at an eGFR $<30 \mathrm{~mL} / \mathrm{min} / 1.73 \mathrm{~m}^{2}$, and therefore metformin should not be administrated in this context because of the risk of overdosing (23). Although several studies have noted the safety of metformin in patients with end-stage renal disease (24), there are no strict evidence approving the beneficial role of metformin in patients under dialysis or those with renal failure.

\section{Dosing recommendations}

So far, there are few evidence on the role of metformin in other solid organ transplant groups. The pathogenesis, diagnosis and management of PTDM are largely unknown and need more investigations. There have been suggestions to adjust the dose of metformin in patients with mild-to-moderate renal damage (i.e. $30<\mathrm{eGFR}<60$ $\mathrm{mL} / \mathrm{min} / 1.73 \mathrm{~m}^{2}$ ) (25). Considering the strong linear correlation between plasma metformin levels and renal function, the dosage of metformin could theoretically be adjusted based on the level of renal function in patients with kidney failure or transplanted individuals to address any safety concern. For drawing an established correlation between metformin levels and renal function; however, one should wait for the reports of in-progress clinical trials (ID: NCT00767351) $(26,27)$. However, there will be a need for extra approvals in renal transplant patients as these patients are more vulnerable to concomitant diseases and therefore need more caution (28).

\section{Evidence}

In 2008, Kurian et al in a small retrospective study examined 32 renal transplant candidates with either pretransplant (number $=11$ ) or post-transplant (number = 21) diabetes administered with either metformin or thiazolidinedione for a period of at least 16 months. There was no significant association between metformin treatment and neither lactic acidosis nor hemoglobin A1c level. However, estimated glomerular filtration rate (eGFR) reduced in patients treated with metformin (29).

In another comprehensive systematic review on 47000 renal transplant candidates in 2001-2012 recruiting the Scientific Registry of Transplant Recipients (SRTR) data, the rate of metformin usage was assessed in renal transplant candidates. In the recent report, the survival of patients received metformin was higher (10\%) than the patients treated with non-metformin regimens; nevertheless, nearly $40 \%$ of the patients treated with metformin had eGFR below the level recommended by the Food and Drug Administration. Moreover, there were no differences in the risk of lactic acidosis and survival rate comparing patients administrated with metformin and non-metformin regimens proposing that metformin did not harmfully affect the survival in transplanted patients (30).

In another retrospective study on 14144 patients with PTDM who received either metformin or insulin, a significant reduction in all-cause mortality and a nonsignificant decline in graft rejection rate were observed in metformin receivers. It is recommended to further scrutinize the drug histories to verify the best and most effective first line anti-diabetes therapy in patients with PTDM (5). In a pilot 1-year randomized clinical trial to investigate the applicability, efficiency, safety, and tolerability of metformin in patients with either prediabetes or PTDM, the effects of metformin were assessed on lipid profile, weight, glycated hemoglobin, fasting glucose and the risk of cardiovascular events. Until today, no comparative trials have been performed on the role of metformin in transplant candidates. According to the Northern B Health and Disability Ethics Committee 
of the Ministry of Health in New Zealand, metformin is a safe and tolerable drug within one year after renal transplantation encouraging clinicians to administer this drug to renal transplant candidates. Nonetheless, this conclusion was based on a non-blinded clinical trial (3), and therefore it is suggested to explore the applicability of metformin in placebo randomized trials.

\section{Conclusion}

More clinical trials are warranted to investigate the role of metformin on the development of PTDM in high-risk renal transplant patients and determine the cost-effective balance of using this drug. Overall, metformin can alleviate dysregulated glucose metabolism and help to both prevent and treat post-transplant diabetes. Metformin also has beneficial effects on weight loss and ameliorates the signs of metabolic syndrome such as insulin resistance and inflammation. Other effects of metformin include lipidlowering impacts, as well as cardio-protective and anticancer properties. Whether or not the beneficial effects of metformin can be extrapolated to high-risk recipients of kidney allografts should be further investigated. One of the potential complications of metformin deterring clinicians to use this drug includes lactic acidosis which its risk is hypothetically exaggerated in allograft recipients. Although there are currently insufficient experimental and clinical evidence, a robust discussion has been declared on the effectiveness and safety of metformin as the firstline anti-diabetes drug in transplant recipients, however, more studies are required to verify the applicability of metformin in this clinical setting.

\section{Authors' contribution}

MA, SHM and AHA participated in the conception of the study, data collection and analysis, and drafted the manuscript. MA, SHM and AHA contributed to drafting, critical reviewing, and finalization of the manuscript. All authors contributed to development of the manuscript and approved the final manuscript.

\section{Conflicts of interest}

The authors declared no competing interests.

\section{Ethical considerations}

Ethical issues including plagiarism, double publication, and redundancy have been completely observed by the authors.

\section{Funding/Support}

None.

\section{References}

1. Piotti G, Gandolfini I, Palmisano A, Maggiore U.Metabolic risk profile in kidney transplant candidates and recipients. Nephrol Dial Transplant. Nephrol Dial Transplant. 2019;34:388-400. doi: 10.1093/ndt/gfy151.
2. Kim YC, Shin N, Lee S, Hyuk H, Kim YH, Kim H, et al. Effect of post-transplant glycemic control on long-term clinical outcomes in kidney transplant recipients with diabetic nephropathy: A multicenter cohort study in Korea. PLoS One. 2018;13:e0195566. doi: 10.1371/journal. pone. 0195566 .

3. Basil Alnasrallah B, Pilmore H, Manley P. Protocol for a pilot randomized controlled trial of metformin in prediabetes after kidney transplantation: the Transplantation and Diabetes (Transdiab) study. BMJ Open. 2017;7:e016813. doi:10.1136/bmjopen-2017-016813.

4. Hecking M, Kainz A, Werzowa J, Haidinger M, Döller D, Tura A, et al. Glucose Metabolism After Renal Transplantation. Diabetes Care. 2013;36:2763-71. doi: $10.2337 / \mathrm{dc} 12-2441$

5. Vest LS, Koraishy FM, Zhang Z, Lam NN, Schnitzler MA, Dharnidharka VR, et al. Metformin use in the first year after kidney transplant, correlates, and associated outcomes in diabetic transplant recipients: A retrospective analysis of integrated registry and pharmacy claims data. Clin Transplant. 2018;32:e13302. doi: 10.1111/ctr.13302.

6. Davidson J, Wilkinson A, Dantal J, Dotta F, Haller H, Hernández D, etal. New-onset diabetes after transplantation: 2003 International consensus guidelines. Proceedings of an international expert panel meeting. Transplantation. 2003;75:SS3-24. doi: 10.1097/01.TP.0000069952.49242.3E.

7. Sharif A, Hecking M, de Vries AP, Porrini E, Hornum $\mathrm{M}$, Rasoul-Rockenschaub S, et al. Proceedings from an international consensus meeting on posttransplantation diabetes mellitus: recommendations and future directions. Am J Transplant. 2014;14:1992-2000.

8. Li J, Gui Y, Ren J, Liu X, Feng Y, Zeng Z, et al. Metformin protects against cisplatin-induced tubular cell apoptosis and acute kidney injury via AMPK alpha-regulated autophagy induction. Sci Rep. 2016;6:23975. doi: 10.1038/srep23975.

9. Lee SY, Moon SJ, Kim EK, Seo HB, Yang EJ, Son HJ, et al. Metformin suppresses systemic autoimmunity in Roquin(san/san) mice through inhibiting B cell differentiation into plasma cells via regulation of AMPK/ mTOR/STAT3. J Immunol. 2017;198:2661-70. doi: 10.4049/ jimmunol.1403088.

10. Zarrinpar A, Bensinger SJ. The therapeutic potential of T cell metabolism. Am J Transplant. 2017;17:1705-12. doi: 10.1111/ajt.14149.

11. Lalau JD, Arnouts P, Sharif A, De Broe ME. Metformin and other antidiabetic agents in renal failure patients. Kidney Int. 2015;87:308-322. doi: 10.1038/ki.2014.19.

12. Shivaswamy V, Bennett RG, Clure CC, Larsen JL, Hamel FG. Metformin improves immunosuppressant induced hyperglycemia and exocrine apoptosis in rats. Transplantation. 2013;95:280-284. doi: 10.1097/ TP.0b013e318275a322.

13. Jin J, Lim SW, Jin L, Yu JH, Kim HS, Chung BH, et al. Effects of metformin on hyperglycemia in an experimental model of tacrolimus- and sirolimusinduced diabetic rats. Korean J Intern Med. 2017;32:314-22. doi: 10.3904/kjim.2015.394.

14. Vanhove T, Remijsen Q, Kuypers D, Gillard P. Drug-drug interactions between immunosuppressants and antidiabetic drugs in the treatment of post-transplant diabetes mellitus. Transplant Rev (Orlando). 2017;31:69-77. doi: 10.1016/j. 
trre.2016.09.001.

15. Sharif A. Should metformin be our antiglycemic agent of choice post-transplantation? Am J Transplant. 2011;11: 1376-1381.

16. Sharif A. Metformin safety post transplantation-trials and tribulations. Am J Transplant. 2012;12:796-796.

17. Larsen JL. Potential risks of metformin in transplant patients. Am J Transplant. 2012;12:795-796. doi: 10.1111/j.1600-6143.2011.03921.x.

18. Shivaswamy V, Boerner B, Larsen J. Post-transplant diabetes mellitus: causes, treatment, and impact on outcomes. Endocr Rev. 2016;37:37-61. doi: 10.1210/er.2015-1084.

19. Salpeter SR, Greyber E, Pasternak GA, Salpeter Posthumous EE. Risk of fatal and nonfatal lactic acidosis with metformin use in type 2 diabetes mellitus. Cochrane Database Syst Rev. 2010:CD002967.doi:10.1002/14651858.CD002967.pub3.

20. Bodmer M, Meier C, Krahenbuhl S, Jick SS, Meier CR. Metformin, sulfonylureas, or other antidiabetes drugs and the risk of lactic acidosis or hypoglycemia: a nested casecontrol analysis. Diabetes Care. 2008;31:2086-91. doi: 10.2337/dc08-1171.

21. Rachmani R, Slavachevski I, Levi Z, Zadok B, Kedar Y, Ravid M. Metformin in patients with type 2 diabetes mellitus: reconsideration of traditional contraindications. Eur J Intensive Care Med. 2002;13:428.

22. Hwang SJ, Lee E, Lee Y-H. Tissue hypoxic condition, not metformin, induces lactic acidosis in patient type 2 diabetes. Presented at: 50th EASD annual meeting 2014, Vienna, Austria; Sept 19th 2014. Diabetologia 57: S100-S100.

23. Sambol NC, Chiang J, Lin ET, Goodman AM, Liu CY, Benet LZ, et al. Kidney function and age are both predictors of pharmacokinetics of metformin. J Clin Pharmacol. 1995;35:1094-102.

24. Al-Hwiesh AK, Abdul-Rahman IS, El-Deen MA, Larbi E, Divino-Filho JC, Al-Mohanna FA, et al. Metformin in peritoneal dialysis: a pilot experience. Perit Dial Int. 2014; 34:368-75. doi: 10.3747/pdi.2013.00048.

25. Lipska KJ, Bailey CJ, Inzucchi SE. Use of metformin in the setting of mild-to-moderate renal insufficiency. Diabetes Care. 2011;34:1431-7. doi: 10.2337/dc10-2361.

26. Lalau JD, Lacroix C, Compagnon P, de Cagny B, Rigaud JP, Bleichner G, et al. Role of metformin accumulation in metformin-associated lactic acidosis. Diabet Care. 1995;18: 779-784.

27. Frid A, Sterner GN, Löndahl M, Wiklander C, Cato A, Vinge E, et al. Novel assay of metformin levels in patients with type 2 diabetes and varying levels of renal function: clinical recommendations. Diabetes Care. 2010;33:1291-3. doi: $10.2337 / \mathrm{dc} 09-1284$.

28. Alangaden GJ, Thyagarajan R, Gruber SA, Morawski K, Garnick J, El-Amm JM, et al. Infectious complications after kidney transplantation: current epidemiology and associated risk factors. Clin Transplant .2006;20:401-9. doi: 10.1111/j.1399-0012.2006. 00519.x

29. Kurian B, Joshi R, Helmuth A. Effectiveness and longterm safety of thiazolidinediones and metformin in renal transplant recipients. Endocr Pract. 2008;14:979-84. doi: 10.4158/EP.14.8.979

30. Stephen J, Anderson-Haag TL, Gustafson S, Snyder JJ, Kasiske BL, Israni AK. Metformin use in kidney transplant recipients in the United States: an observational study. Am J Nephrol. 2014;40:546-53. doi: 10.1159/000370034.

Copyright $\odot 2019$ The Author(s); Published by Nickan Research Institute. This is an open-access article distributed under the terms of the Creative Commons Attribution License (http://creativecommons.org/licenses/by/4.0), which permits unrestricted use, distribution, and reproduction in any medium, provided the original work is properly cited. 